\title{
TECHNOLOGY OF NON DESTRUCTIVE CHECK UP IN INDUSTRY
}

\author{
George Kurilenko, Michael Sazonov, Nicolay Troitsky \\ Novosibirsk State Technical University, K. Marx Av., 20,,630092, \\ Novosibirsk, Russia \\ Tel.: 0073832461777 Fax: 0073832460301 E-mail: \\ art@mail.fam.nstu.ru
}

Keywords: crack resistance, non destructive check, thermographic approach, entropy.

Use thermographic parameters of fatigue development becomes possible realization of actual engineering problem, namely: manages or not given real detail to work indented cyclic lifetime.

At first, we defined the level of control stress $\sigma_{c}$. For that purpose the individual fatigue limits $\sigma_{r}$ for some details (control part) of given technological level production are defined. After that we made statistical treatment of received results and as the control stress $\sigma_{c}$ accept the lower level of fatigue limit, calculated with given confidential probability (as a rule, it is accepted $95^{\text {th }} \%$ or $90^{\text {th }} \%$ confidential probability).

Then we test real detail on the level $\sigma_{c}$ (the time of cyclic loading is very small) and calculate the increment specific entropy $\Delta S^{1 c}$ for one cycle of oscillation by the formula:

$$
\Delta S^{1 c}=c_{v} \ln \frac{T_{2}}{T_{1}},
$$

where $c_{v}$ is the specific heat capacity of material;

$T_{2}$ and $T_{1}$ are the temperatures on the surface of the domain of damaging at the end and beginning of a particular cycle.

After that we test on the level $\sigma_{c}$ those details from control part which have individual fatigue limits $\sigma_{r}$ not less than $\sigma_{c}$

$$
\sigma_{r} \geq \sigma_{c}
$$


and calculate the values $\Delta S^{1 c}$ by formula (1).

As a result the critical value of increment specific entropy for one cycle of oscillation $\Delta S_{c r}^{1 c}$ is defined as upper statistical level $\Delta S^{1 c}$.

The real detail recognizes suitable if after its testing on the level of control stress $\sigma_{c}$ we have:

$$
\Delta S^{1 \mathrm{c}} \leq \Delta S_{c r}^{1 c}
$$

The method described has some advantages in comparison with traditional approaches. These advantages come from its higher accuracy, efficiency and universal applicability.

The practical implementation of the proposed thermographic method could be made using up-to-date infrared equipment.

This method is successfully used in the inspection of cyclic durability of real parts: shuttle springs and torsion shafts of loom. 